ARTICLE OPEN

\title{
Cleavage-dissolution assisted stress corrosion cracking under elastic loads
}

\author{
Longkui Zhu $\mathbb{D}^{1 凶}$ and Yao $\mathrm{Li}^{1}$
}

As a significant cause of disastrous accidents, stress corrosion cracking (SCC) under elastic loads was investigated in type $316 \mathrm{~L}$ single-crystal stainless steel immersed in a boiling $45 \mathrm{wt} \% \mathrm{MgCl}_{2}$ solution. Three-dimensional microcrack morphologies, characterized using synchrotron-based X-ray computed tomography, indicate that the SCC advanced along the cleavage planes

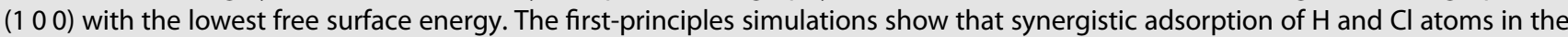

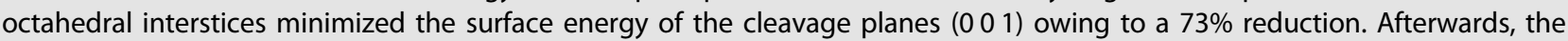
cleavage-dissolution mechanism is put forward, proposing that the SCC essentially originates from preferential brittle rupture of the corrosive environment particle adsorbed low-surface-energy cleavage planes in the elastic stress concentration zones, and anodic dissolution along the crack fronts. Besides, the corrosive environment particles primarily consist of the hydrogen atoms and the electronegative ions such as the chlorine ions.

npj Materials Degradation (2021)5:25; https://doi.org/10.1038/s41529-021-00172-7

\section{INTRODUCTION}

Documentary phenomena of metal-crystal stress corrosion cracking (SCC) in chloride solutions over the past decades indicate that the SCC crack advance emanates from localized dissolution within crack tips and rupture of crystal planes or grain boundaries at the scales of the microns ${ }^{1-10}$. It is considered that passive films due to the dissolution of metal anodes are broken to induce the SCC growth under the action of slip bands ${ }^{1,5}$. Qiao et al. ${ }^{2,6}$ demonstrate that the SCC cracks unnecessarily nucleate and propagate along slip planes or surface slip bands. Numerous experimental results unmask that the SCC is apparently related with cleavage planes as well as pitting at the low-stress levels ${ }^{7-10}$.

Various microscopic mechanisms on SCC have been put forward until now. Among them, the slip-dissolution model is considered by Newman and Healey ${ }^{11}$ as an important milestone in corrosion science. It is illustrated that an SCC process can be divided into three sequential stages, including dissolution of fresh slip planes as anodes, formation of passive films, and film rupture by slipping ${ }^{1,5,11-15}$. Namely, SCC initiation and propagation in the chloride solutions are induced by corrosion of the slip planes and cracking of the films on the fresh planes. Afterwards, the corrosion-enhanced plasticity model has also been put forward, to primarily elucidate hydrogen-dislocation interactions for the metal-crystal SCC in the chloride solutions. It is proposed that the local stresses increase due to dislocation pile-ups partially induced by solute hydrogen, while on crystal planes $\left\{\begin{array}{lll}1 & 1 & 1\end{array}\right\}$ or $\left\{\begin{array}{lll}1 & 0 & 0\end{array}\right\}$ the critical stress intensity factors, $K_{\mathrm{I}}$, decrease simultaneously owing to hydrogen as well ${ }^{16-20}$. In the above two SCC models, slip bands, as well as plastic deformation, are hypothetically acted as the essential SCC features, because of the observed experimental phenomena that the SCC was accompanied by dislocation slipping under high loads, such as in the slow strain rate tensile tests ${ }^{17}$ and the U-shaped bending tests ${ }^{21}$. However, even if at the high stress levels, numerous microscopic SCC cracks did not actually advance along the slip planes $\left\{\begin{array}{lll}1 & 1 & 1\end{array}\right\}$ or the surface slip bands in the single and poly crystals ${ }^{2,6,9}$. Under low loads, the SCC cleavage cracks, coinciding with the river-like fractography, also nucleated and propagated along the cleavage planes without the occurrence of the dislocation slipping ${ }^{8,9}$. Besides, on the basis of the molecular statics simulations, the rupture of the grain boundary was accompanied by dislocation emission without hydrogen, while the cleavage without dislocations nor amorphization occurred under the action of hydrogen atoms in the facecentered cubic bicrystals ${ }^{22}$. These convincing experimental and simulative phenomena as mentioned above manifest that the SCC is a kind of brittle fracture on the cleavage planes subjected to the ultra-low normal stresses, rather than a ductile process induced by the dislocation slipping at the high stress levels.

Moreover, it is well known that anodic dissolution of the fresh cracking surfaces can promote SCC advance in the chloride solutions, at least through stress concentration along SCC crack fronts ${ }^{7,8,10}$, together with adsorption of electronegative ions and hydrogen atoms on the crystal planes ${ }^{18,23-26}$. Obviously, the acidic chloride solutions inside the SCC crack tips were in favor of the localized corrosion attack on the crack fronts in the open-circuit conditions $^{27,28}$, and the corrosion product films did not tend to be formed during the SCC growth processes ${ }^{3}$. Even though the nanometer-thick films cover the matrix surfaces, the chlorine ions were able to attack and break down the passive films, detected by the transmission electron microscopy $(\text { TEM })^{26}$. Thus, the anodic dissolution could take place on the fresh metal surfaces. Sometimes, some microscopic dissolved pits emerged along the crack fronts, and the tensile stresses concentrated at the pit shoulders ${ }^{8}$. Further, it is considered that electronegative ions and hydrogen atoms, originating from the electrochemical reactions, are able to act on the crystal planes and the atoms on front of the SCC crack tips. The unified model of environment-assisted cracking proposes that valence electrons of these chemicals and the hydrogen atoms lower the misfit energy and the resistance of slipping and dislocation motion ${ }^{23,29}$. Nevertheless, the atom probe tomography observation of hydrogen at dislocations and grain boundaries directly provided validation for hydrogen embrittlement ${ }^{30}$. The

\footnotetext{
'Beijing Institute of Structure and Environment Engineering, China Academy of Launch Vehicle Technology, China Aerospace Science and Technology Corporation, Beijing, China. 凶email: I.k.zhu@163.com
} 
multiscale simulations and subsequent analysis suggest that electronegative impurities could inhibit dislocation nucleation from a loaded crack tip in face-centered cubic aluminum in the presence of hydrogen and oxygen ${ }^{31}$. Besides, the TEM detection and the atomic simulation show that the permeated chlorine also induced lattice expansion of the metal matrix along the crystal direction $\left[\begin{array}{lll}1 & 0 & 0\end{array}\right]^{26}$.

From the perspective of the brittle fracture and the localized dissolution, this article first analyzes three-dimensional SCC crack morphologies and crystallographic features under elastic loads, along with the surface energy of $\mathrm{H}$ - and $\mathrm{Cl}$-adsorbed cleavage planes. Afterwards, a non-slipping SCC model is proposed to elucidate essential electrochemical and physical mechanisms, including interactions of corrosive environment particles with crystal planes, brittle rupture of low-surface-energy cleavage planes and so on.

\section{RESULTS AND DISCUSSION}

\section{Three-dimensional SCC crack morphologies and crystallographic analysis}

The two- and three-dimensional crack morphologies along with the crystallographic features were characterized to analyze the elastic-load SCC cracks of the type $316 \mathrm{~L}$ single-crystal stainless steel immersed in the boiling $45 \% \mathrm{MgCl}_{2}$ solution, shown in Fig. 1. No slip bands emerged on the specimen surfaces. Both the superficial and the internal SCC cracks advanced along the parallel crystal planes in a zig-zag manner, coinciding with the river-like fractography $^{8,9}$. Simultaneously, the Euler angles $\left(\varphi_{1}, \Phi, \varphi_{2}\right)$ were measured as $\left(317.8^{\circ}, 10.3^{\circ}\right.$, and $\left.18.9^{\circ}\right)$ around the SCC cracks. The angle of the original crystal direction $\left[u_{0} v_{0} w_{0}\right]$ and the SCC propagation direction $[u v w]$ was $60^{\circ}$, while the angle of the original crystal plane $\left(h_{0} k_{0} I_{0}\right)$ and the SCC cleavage plane $(h k l)$ was $103^{\circ}$. On the basis of the two-surface trace analysis, the cleavage planes $(h k l)$ were the crystal planes $(100)$ with the lowest free surface energy, rather than the slip planes $\left\{\begin{array}{lll}1 & 1 & 1\end{array}\right\}$. These experimental results convincingly demonstrate that the nonslipping SCC could initiate and propagate along the cleavage planes. It is also apparent that these novel elastic-load SCC phenomena are obviously distinct from the previous SCC mechanisms, such as the slip-dissolution model ${ }^{1,5,11-15}$, the corrosion-enhanced plasticity model ${ }^{16-20}$, the film induced cleavage model ${ }^{32-34}$, and the uniform environment-assisted cracking model $^{23,29}$.

\section{Surface energy of $\mathbf{H}$ - and $\mathrm{Cl}$-adsorbed cleavage planes}

Since the type $316 \mathrm{~L}$ stainless steel was roughly equivalent to the face-centered cubic $\mathrm{Fe}_{5} \mathrm{Cr}_{2} \mathrm{Ni}$ alloy, the $\mathrm{Fe}_{5} \mathrm{Cr}_{2} \mathrm{Ni}$ slabs with and without $\mathrm{H}$ or $\mathrm{Cl}$ adsorption on the cleavage surfaces $\left(\begin{array}{lll}0 & 0 & 1\end{array}\right)$ were optimized through the density functional theory (DFT) in the VASP code, shown in Fig. 2. The $\mathrm{H}$ and $\mathrm{Cl}$ atoms were homogeneously distributed in the octahedral interstices of the top layer, and the lattice distortion occurred on the adsorbed surfaces. The firstprinciples calculations indicate that the free surface energy of the slab, $\gamma_{(001)}$, the $\mathrm{H}$-adsorbed surface energy of the slab with $4 \mathrm{H}$ atoms, $Y_{(001)}^{\mathrm{H}}$, and the $\mathrm{H}$ - and $\mathrm{Cl}$-adsorbed surface energy of the slab with $2 \mathrm{H}$ atoms and $2 \mathrm{Cl}$ atoms, $\mathrm{Y}_{(001)}^{\mathrm{H}+\mathrm{Cl}}$, were separately estimated to be $3.07,2.21$, and $0.84 \mathrm{~J} \mathrm{~m}^{-2}$. Compared with $\gamma_{(001)}$, $\gamma_{(001)}^{\mathrm{H}}$ was reduced by $0.86 \mathrm{~J} \mathrm{~m}^{-2}$ and $28 \%$, while $\gamma_{(001)}^{\mathrm{H}+\mathrm{Cl}}$ even decreased by $2.23 \mathrm{~J} \mathrm{~m}^{-2}$ and $73 \%$. Furthermore, in the light of the Griffith's theory, $K_{\mathrm{IC}}$ of the cleavage planes $\left(\begin{array}{ll}0 & 0\end{array}\right)$ was lowered by $15 \%$ and $48 \%$ in the conditions of $\mathrm{H}$ adsorption and $\mathrm{H}$ combined with $\mathrm{Cl}$ adsorption, respectively. It is manifested that only the $\mathrm{H}$ adsorption was weak or not sufficient, but the synergistic effects of $\mathrm{H}$ and $\mathrm{Cl}$ adsorption were fairly intense, which might significantly induce the brittle rupture of the cleavage planes $\left(\begin{array}{lll}0 & 0 & 1\end{array}\right)$ at the ultra-low elastic stress levels.

\section{Modeling of the elastic-load SCC}

The cleavage-dissolution model is proposed on the basis of the above experimental and simulative results as follows: SCC initiation and propagation emanate from synergistic effects of a brittle rupture occurring on low-surface-energy cleavage planes in elastic stress concentration zones (ESCZs) on front of the crack tips, and anodic dissolution of microscopic electric couples along the SCC crack fronts. During the SCC processes, the original and generated electronegative ions, hydrogen atoms, or vacancies are chemically or physically interacted with the cleavage planes, leading to the decrease of their surface energy. In addition, the embrittlement of the ESCZs is attributed to the pinning of the intrinsic dislocations under the actions of the electronegative ions and the hydrogen atoms. Thus, the SCC cracks preferentially advance along the environment-assisted low-surface-energy crystal planes, not simultaneously accompanied by slipping or dislocation motion under the low loads.

Figure 3 schematically depicts the sequential SCC steps. Above all, a chloride solution permeates an SCC crack tip in the light of the capillary effects. The second step is anodic dissolution of microscopic electric couples with potential differentials, due to heterogeneity of chemical compositions, stress distribution on metal surfaces, ion density inside the SCC crack tip, and so on. Meanwhile, an ESCZ is formed around the SCC crack front. Some original and generated electronegative ions, hydrogen atoms, or vacancies are interacted with crystal planes in the ESCZ, decreasing their surface energy as well as their cohesive forces. Afterwards, if the concentrated normal stress applied to a lowsurface-energy cleavage plane amounts to its decreased critical cleavage stress, the cleavage plane occurs to rupture, and a fresh
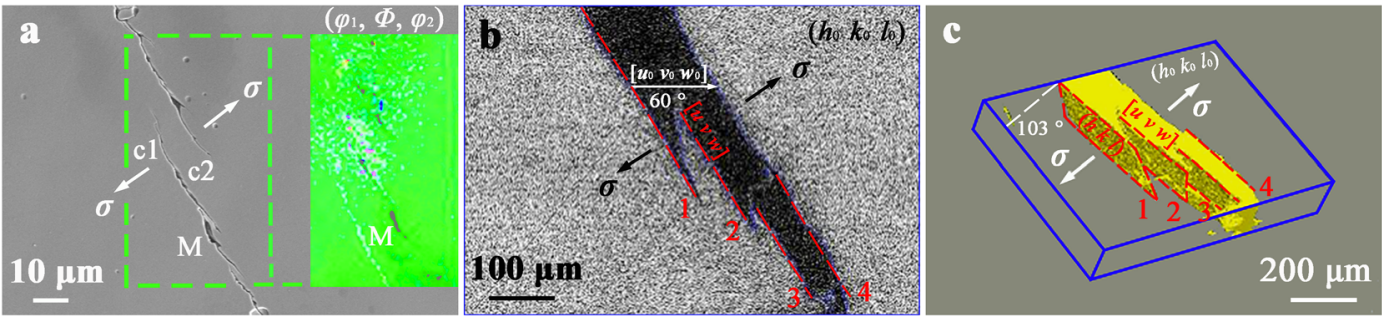

Fig. 1 Non-slipping SCC on cleavage planes $\left(\begin{array}{lll}1 & 0 & 0\end{array}\right)$. a The SEM morphologies of the surface cracks ' $c 1$ ' and ' $c 2$ ', along with the dotted-greenrectangle and ' $\mathrm{M}$ '-marked surface crystallography corresponding to the Euler angle $\left(\varphi_{1}, \Phi, \varphi_{2}\right)=\left(317.8^{\circ}, 10.3^{\circ}, 18.9^{\circ}\right)$ characterized by the EBSD. $\mathbf{b}$ The XCT morphologies of a surface-parallel crossing section with the Euler angle $\left(\varphi_{1}, \Phi, \varphi_{2}\right)=\left(317.8^{\circ}, 10.3^{\circ}, 18.9^{\circ}\right)$, the original crystal plane $\left(h_{0} k_{0} I_{0}\right)$, the original crystal direction $\left[u_{0} v_{0} w_{0}\right]$, and the SCC propagation direction [uvw], in which the angle of [ $\left.u_{0} v_{0} w_{0}\right]$ and [uv $\left.w\right]$ is $60^{\circ}$, and the crack edges and the cleavage plane edges are indicated by the dotted red lines ' 1 ', ' 2 ', ' 3 ' and ' 4 '. c The partial through-thickness XCT morphologies of the SCC cracks with the cleavage planes $(h \mathrm{kl})$, in which the angle of $\left(h_{0} k_{0} I_{0}\right)$ and $(h k I)$ is $103^{\circ}$, and the crack edges are also indicated by the dotted red lines ' 1 ' to ' 4 ', and the SCC cracks are marked by yellow. The calculations show that ( $h k l)$ was $(100)$, and the SCC advanced along the cleavage planes $\left(\begin{array}{lll}1 & 0 & 0\end{array}\right)$. 
$\mathbf{a}$

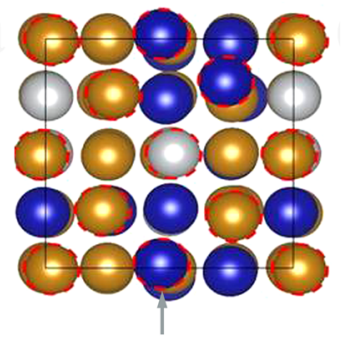

b

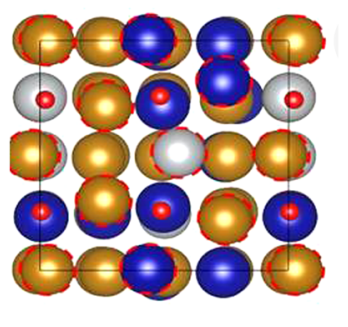

c

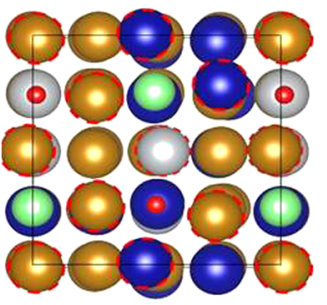

d

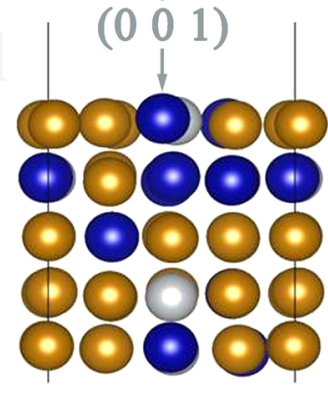

e

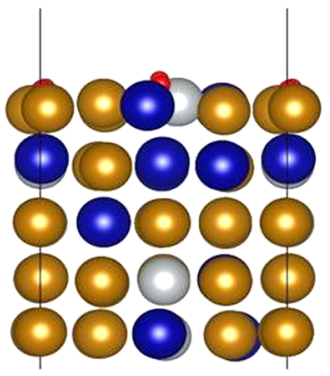

f

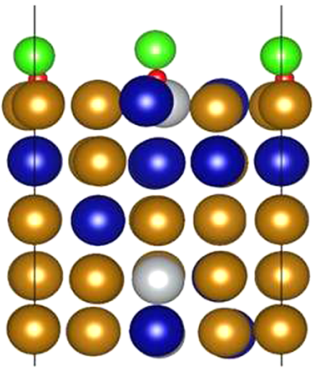

Fe

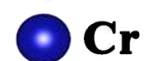

$\mathbf{a}$

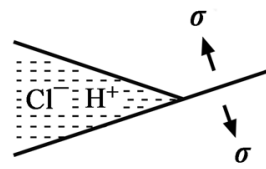

Low-Surface-Energy Crystal Plane

b

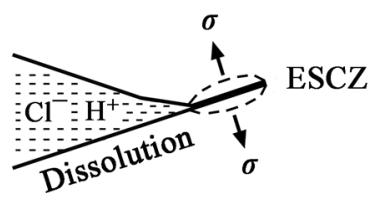

c

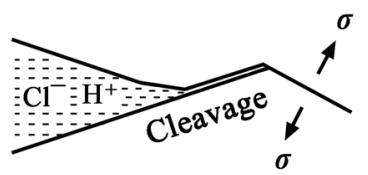

d

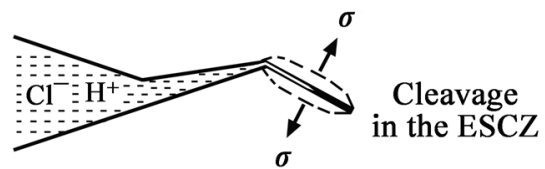

Fig. 3 A typical cleavage and dissolution process of SCC. a The anodic dissolution of the microscopic electric dipoles first occurs at the crack tip, promoting the SCC advance and the stress concentration. b An ESCZ is formed on front of the crack tip, in which the corrosive environment particles interact with the crystal planes and pin the intrinsic dislocations. c A corrosive environment particle

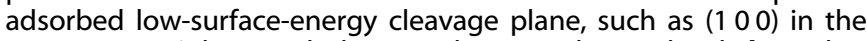
type $316 \mathrm{~L}$ stainless steel, cleave at the normal stress level. d Another SCC microcrack nucleates through repetition of anodic dissolution, forming another ESCZ and brittle rupture of another low-surfaceenergy cleavage plane.

SCC microcrack nucleates in the ESCZ, which induces SCC growth at a little distance toward. In this way, another fresh SCC microcrack is capable of nucleation, through repetition of anodic dissolution, formation of another $\mathrm{ESCZ}$, and rupture of another low-surface-energy cleavage plane, schematically shown in the final step in Fig. 3d.
Interactions of corrosive environment particles with the ESCZs The corrosive environment particles primarily consist of the electronegative ions and the hydrogen atoms. As usual, the electronegative ions along with the vacancies are generated owing to the anodic dissolution on the SCC crack fronts ${ }^{23,35}$, while the hydrogen cations in the chloride solutions were reduced as the hydrogen atoms on the cathode surfaces ${ }^{23,27,28}$. Although the radii of the octahedral interstice in the type $316 \mathrm{~L}$ austenitic stainless steel, the hydrogen atom and the chlorine atom are separately $0.019,0.053$, and $0.099 \mathrm{~nm}$, these generated corrosive environment particles and vacancies, together with the original ions in the solutions and the original vacancies inside the metal matrixes, are capable of diffusion in the ESCZs, pinning the dislocations and adsorption with the cleavage planes, shown in Fig. 4.

In the light of the Fick's law, the corrosive environment particles permeate into the microscopic defects such as vacancies, interstices, dislocations, and voids, or sometimes replacing the metal atoms in the ESCZs. The diffusion flux, $J$, of a corrosive environment particle, $i$, under the concentrated tensile stress is ${ }^{36}$ :

$J=J_{c}+J_{\sigma}=-D_{i} \frac{\partial C_{i}}{\partial x_{i}}+\frac{D_{i} C_{i} V_{i}}{R T} \frac{\partial \sigma_{i}}{\partial x_{i}}$

where $J_{c}$ and $J_{\sigma}$ are the diffusion fluxes induced by the density differential and the tensile stress, respectively, $D_{i}$ is the diffusion coefficient of the corrosive environment particle, $C_{i}$ is the corrosive environment particle density, $x_{i}$ is the diffusion distance of the corrosive environment particle, $V_{i}$ is the volume change owing to the spherical-symmetry strain field induced by the corrosive environment particle, and $\sigma_{i}$ is the tensile stress in the sphericalsymmetry strain field, $R$ is the gas constant, $R=8.314 \mathrm{~J} \mathrm{~mol}^{-1} \mathrm{~K}^{-1}$, and $T$ is the Kelvin temperature. In this way, the corrosive environment particles are able to keep on moving forward in the ESCZs. In addition, the vacancies in the ESCZs are apt to diffuse along the stretched crystal planes.

Obviously, some of the corrosive environment particles during the diffusion processes are probably trapped into vacancies, dislocations, or voids in the ESCZs, shown in Fig. 4b. Once a dislocation is pinned by a corrosive environment particle, the 

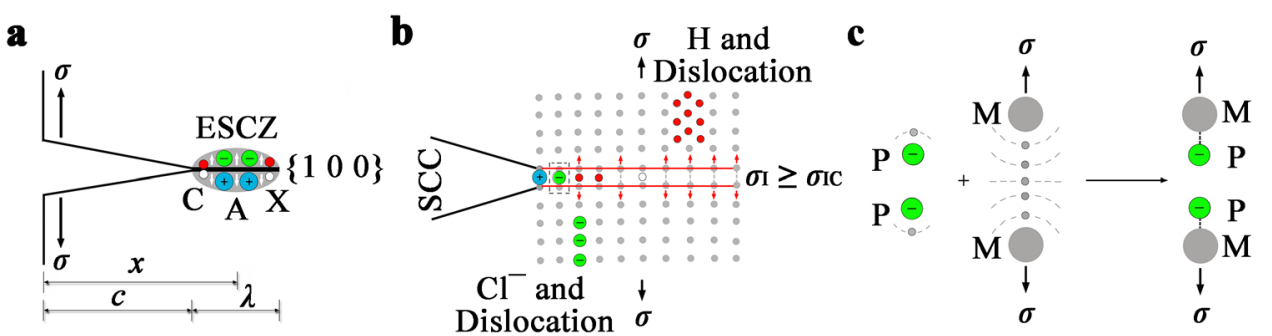

Fig. 4 An atomic-scale brittle rupture and dislocation pinning model. a In the ESCZ, cations, anions, hydrogen atoms, and vacancies are marked by green, blue, red, and white circles, respectively. b The SCC advance along the corrosive environment particle adsorbed low-surfaceenergy cleavage planes such as $\{100\}$ on $\sigma_{1} \geq \sigma_{\mathrm{IC}}$, and the intrinsic dislocations in the ESCZ are pinned by the hydrogen atoms and the electronegative ions such as the chlorine ions. c The chemical and physical interactions between the metal atoms on the cleavage planes and the microscopic corrosive particles, $P$, such as the electronegative ions, marked by the dotted rectangle in (b).

corresponding slip system is prevented to be triggered, which increases the critical shear stress and the resistance of the dislocation slipping ${ }^{24,25}$. For instance, the hydrogen atoms are able to weaken the pair interactions between the coplanar dislocations ${ }^{18,20}$, and lower the effective speed, at which the dislocation loop shrinks ${ }^{25}$. Similarly, the other solute atoms can also pin the dislocations and prohibit the dislocation motion through the formation of the Cottrell or Snoek atmospheres. To sum up, the pinning of the dislocations by the corrosive environment particles leads to the embrittlement of the ESCZs in the ductile metal crystals.

Further, the electronegative ions, the hydrogen atoms, and the vacancies are interacted with the metal atoms of the cleavage planes in the ESCZs, shown in Fig. 4b, c. For instance, the $1 \mathrm{~s}$ orbital electrons of the hydrogen atoms are generally migrated to the metal atom orbital ' $d$ ', so the metal atoms are full of electrons and become saturated ${ }^{23,35}$. To some extent, the electron rearrangement weakens the metallic bonds between the cleavage planes. Second, the distribution of the $\mathrm{H}$ and $\mathrm{Cl}$ atoms in the octahedral interstices causes the crystal lattices to be deformed elastically, leading to the increase of the atomic distances and the decrease of the cohesive forces between the atoms. Third, the $\mathrm{H}$ and $\mathrm{Cl}$ atoms are capable of chemical and physical adsorption with the metal atoms to decrease the free surface energy of the cleavage planes, observed in our simulations and the previously reported literatures ${ }^{18,37}$. In addition, the vacancies are able to lower the cohesive forces between the cleavage planes as well.

In short, the free surface energy of the cleavage planes, $\gamma$, is reduced to the environment-assisted surface energy, $\gamma_{\mathrm{e}}$, under the synergistic actions of the electronegative ions, the hydrogen atoms, and the vacancies. That is, $\gamma_{\mathrm{e}}$ can be written as:

$\gamma_{e}=\gamma-W_{e}$

where $W_{\mathrm{e}}$ is named by the environmental work owing to the activated diffusion, the chemical and physical adsorption, and the elastic strain fields of the corrosive environment particles as well as the vacancies on the cleavage planes. Correspondingly, the environmental work is composed of the diffusion activation energy, $W_{D}$, the adsorption energy, $W_{A}$, and the strain energy, $W_{\varepsilon}$. Namely, $W_{\mathrm{e}}$ is:

$W_{\mathrm{e}}=W_{\mathrm{D}}+W_{\mathrm{A}}+W_{\varepsilon}$

At length, the diffusion activation energy, $W_{D}$, induced by $n_{1}$ corrosive environment particles (and vacancies) on the cleavage planes, is given by:

$W_{\mathrm{D}}=R T \sum_{i=1}^{n_{1}} \ln \frac{D_{i 0}}{D_{i}}$

where $D_{i 0}$ is the diffusion constant of each particle or vacancy. In general, $W_{\mathrm{D}}$ is just a little part of $W_{\mathrm{e}}$ and sometimes omitted. Surely the chemical and physical adsorption is considered as one of the crucial interactions of the corrosive environment particles with the cleavage planes. In the light of the Gibbs' adsorption theory, the adsorption energy, $W_{\mathrm{A}}$, is ${ }^{38-40}$ :

$$
W_{\mathrm{A}}=k T \sum_{i=1}^{n_{2}} \int \Gamma_{i}\left(P_{i}\right) d\left(\ln P_{i}\right)
$$

where $n_{2}$ is the number of the corrosive environment particles (and vacancies) on the cleavage planes, $k$ is the Boltzmann constant, $k=8.167 \times 10^{-5} \mathrm{eVK}^{-1}, p_{i}$ is the gas pressure of each particle or vacancy, $\Gamma_{i}\left(p_{i}\right)$ is the concentration of each particle or vacancy on the stretched cleavage plane, which is the function of $p_{i}$. At constant temperature, $\Gamma_{i}\left(P_{i}\right)=\Gamma_{i 0} P_{i}^{\eta}\left(P_{i}^{\eta}+P_{i 0}^{\eta}\right)^{-1}$, where $\Gamma_{i 0}$ is the concentration of each particle or vacancy on the closed cleavage plane, $p_{i 0}$ is the intrinsic gas pressure of each particle or vacancy, and $\eta$ is the probability of each particle or vacancy emerging at the unit area. Besides, the elastic strain energy, $W_{\varepsilon}$ around each particle or vacancy is:

$W_{\varepsilon}=\frac{1}{2} \sum_{i=1}^{n_{3}} F_{i} \Delta_{i}=\frac{1}{2} \sum_{i=1}^{n_{3}} Y d A_{i} \varepsilon_{i}^{2}$

where $n_{3}$ is the number of the corrosive environment particles (and vacancies) on the cleavage planes, $Y$ is the Young's modulus, $d$ is the original distance of the stretched cleavage planes, $A_{i}$ is the elastic deformation area of the stretched cleavage planes, $\varepsilon_{i}$ is the elastic tensile strain induced by the expansion of the crystal lattices, $\Delta_{i}$ is the corresponding length of the elastic tensile deformation, $F_{i}$ is the corresponding elastic tensile stress. Consequently, submitting Eqs. (4-6) into Eq. (3) yields the environmental work acting on the cleavage plane, owing to the diffusion of $n_{1}$ particles (and vacancies) and the adsorption of $n_{2}$ particles (and vacancies), as well as the elastic strains owing to $n_{3}$ particles (and vacancies). That is, $W_{\mathrm{e}}$ can be written as:

$$
W_{e}=R T \sum_{i=1}^{n_{1}} \ln \frac{D_{i 0}}{D_{i}}+k T \sum_{i=1}^{n_{2}} \int \Gamma_{i}\left(P_{i}\right) d\left(\ln P_{i}\right)+\frac{1}{2} \sum_{i=1}^{n_{3}} Y d A_{i} \varepsilon_{i}^{2}
$$

Therefore, the environment-assisted surface energy, $\gamma_{\mathrm{e}}$, also named by the residual surface energy, is able to approach the critical value to fracture the cleavage planes in the ESCZs during the SCC.

\section{Brittle rupture of environment-assisted cleavage planes}

At the elastic stress levels, the non-slipping SCC advances along the environment-assisted low-surface-energy cleavage planes. In principle, this kind of brittle cleavage in the ESCZs coincides with the Griffith's theory. The critical stress intensity factor, $K_{\mathrm{IC}}$, for the SCC initiation along an environment-assisted low-surface-energy cleavage plane can be given by:

$$
K_{\mathrm{IC}}=\sqrt{\frac{2 \gamma_{e}}{1-v^{2}}}
$$


where $v$ is the Poisson's ratio of the metal matrix. In terms of Eq. (2), $K_{\mathrm{IC}}$ is also written as:

$K_{\mathrm{IC}}=\sqrt{\frac{2 Y\left(\gamma-W_{e}\right)}{1-\mathrm{v}^{2}}}$

Correspondingly, the critical normal stress, $\sigma_{I C}$, to fracture the environment-assisted low-surface-energy cleavage plane is:

$$
\sigma_{\mathrm{IC}}=\sqrt{\frac{r Y\left(\gamma-W_{e}\right)}{4 a_{0} c}}
$$

where $a_{0}$ is the balanced atomic distance, $c$ is the SCC crack length, and $r$ is the curvature radius at the SCC crack tip. Thus, both $K_{\mathrm{lC}}$ and $\sigma_{\mathrm{IC}}$ depend on the free surface energy and the environmental work acting on the cleavage planes. Namely, the SCC crack tends to initiate and propagate on the cleavage planes either with the low-surface energy or with the large environmental work, and preferentially advances on the corrosive environment particle adsorbed low-surface-energy cleavage planes. It is demonstrated that the SCC cleavage plane of the austenitic stainless steel is one of the crystal planes $\left\{\begin{array}{lll}1 & 0 & 0\end{array}\right\}$ in the experiments, or sometimes belongs to $\left\{\begin{array}{lll}1 & 1 & 0\end{array}\right\}$ or $\left\{\begin{array}{lll}1 & 1 & 2\end{array}\right\}$ reported in the literatures ${ }^{18,41-45}$

Quantitatively, assuming that the SCC crack propagates by an area, $\Delta A$, in the ESCZ, and the static energy of the applied system is correspondingly reduced by $-\Delta U$, the crack propagation dynamics, $G_{1}$, also named by the system energy release rate in the condition of the unit crack propagation area, is given by:

$G_{l}=-\frac{\Delta U}{\Delta A}=-\frac{\partial U}{\partial A}$

Since there is the elastic deformation in the specimen or the structure subjected to the applied load, the increase of the applied stress work, $\Delta W_{\sigma}$, contains the elastic strain energy increase of the whole specimen or structure, $\Delta W_{E}$, and the kinetic energy of the crack propagation, $G_{l} \Delta A$. Namely, $G_{l}$ can also be written as:

$G_{l}=-\frac{\Delta W_{\mathrm{E}}-\Delta W_{\sigma}}{\Delta A}=-\frac{\partial\left(W_{\mathrm{E}}-W_{\sigma}\right)}{\partial A}$

In the light of Eqs. (9-10), the SCC propagation resistance, $R_{\mathrm{SCC}}$ is:

$R_{\mathrm{SCC}}=\mathrm{\gamma}-W_{\mathrm{e}}$

When $G_{l} \geq R_{\mathrm{SCC}}$, the environment-assisted low-surface-energy cleavage plane ruptures. The cleavage-assisted SCC propagation rate, $v_{\mathrm{c}}$ is given by:

$v_{\mathrm{c}}=a_{0} f_{0} \exp \frac{G \Delta A}{k T}=a_{0} f_{0} \exp \left(-\frac{\Delta W_{\mathrm{E}}-\Delta W_{\sigma}}{k T}\right)$

where $f_{0}$ is the basic lattice frequency, $f_{0}=k T h^{-1}$, and $h$ is the Planck constant, $h=4.136 \times 10^{-15} \mathrm{eV} \cdot \mathrm{s}$. Given that the anodic dissolution promotes the SCC advance, the cleavage-dissolution assisted SCC propagation rate, $v_{\mathrm{SCC}}$, can be written as:

$v_{\mathrm{SCC}}=v_{\mathrm{c}}+v_{\mathrm{a}}=a_{0} f_{0} \exp \left(-\frac{\Delta W_{\mathrm{E}}-\Delta W}{k T}\right)+v_{\mathrm{a}}$

where $v_{\mathrm{a}}$ is the anodic dissolution assisted SCC propagation rate, which is systematically expressed in the section of Supplementary Discussion.

Moreover, although both the critical cleavage stress, $\sigma_{\mathrm{I}}$, and the critical shear stress, $\tau_{\mathrm{C}}$, are simultaneously changed under the actions of the corrosive environment particles and the vacancies, the apparent distinction between the two kinds of environmental effects is $\sigma_{I C}$ deceases on the cleavage planes due to the synergistic effects of the corrosive environment particles ${ }^{18,20,23}$, whereas $\tau_{C}$ increases on the slip planes on account of the dislocation pinning ${ }^{18,20,24,25,30}$. In addition, $\sigma_{\mathrm{IC}}$ is likely to be localized highly on a couple of metal atoms, while $\tau_{C}$ corresponds to numerous atoms of the neighboring slip planes. As a consequence, the brittle cleavage during the SCC is able to preferentially take place without the dislocation slipping at the elastic stress levels.

The main conclusions are:

The two- and three-dimensional morphologies along with the crystallographic analysis of the microscopic SCC cracks indicate that the SCC advanced along the low-surface-energy cleavage planes (1 00$)$ ) without the slip bands on the specimen surfaces. The firstprinciples calculations show that the synergistic adsorption of the $\mathrm{H}$ and $\mathrm{Cl}$ atoms in the octahedral interstices minimized the surface energy of the cleavage planes $(001)$ owing to a $73 \%$ reduction, while the surface energy only decreased by $28 \%$ in the $\mathrm{H}$-adsorbed conditions. On the basis, the cleavage-dissolution model is proposed, considering that the SCC initiation and propagation in metal crystals originate from the synergistic effects of the brittle rupture occurring on the low-surface-energy cleavage planes in the ESCZs, and the anodic dissolution on the microscopic electric couples along the SCC crack fronts. During the SCC processes, the chemical and physical interactions of electronegative ions, hydrogen atoms, vacancies with the low-surface-energy cleavage planes lead to the decrease of their surface energy, and the fracture of some atomic bonds between these cleavage planes. Consequently, the corrosive environment particle adsorbed low-surface-energy cleavage planes, such as $\{100\}$ in the type $316 \mathrm{~L}$ single-crystal stainless steel, can rupture preferentially in the ESCZs, not simultaneously accompanied by the slipping owing to the pinning of the dislocations. The cleavage-dissolution model is apparently distinct from the well-known SCC models such as the slipdissolution model and the corrosion-enhanced plasticity model.

\section{METHODS}

\section{SCC tests and synchrotron-based X-ray computed tomography}

The type $316 \mathrm{~L}$ single-crystal stainless steel were used in this study with the following chemical composition: C-0.007 wt $\%, \mathrm{Cr}-17.00$ wt $\%, \mathrm{Ni}-13.49$ wt $\%$, Mo-2.54 wt \%, Mn-0.66 wt\%, Si-0.46 wt\%, P-0.0080 wt\%, S- 0.0056 wt $\%$, and Febalance. The 0.5 -mm-thick specimens with the maximum stress concentration coefficient, 2.1, subjected to an elastic nominal stress of $20 \mathrm{MPa}$ and applied using a weight-type constant load apparatus, were immersed into a boiling $45 \mathrm{wt} \% \mathrm{MgCl}_{2}$ solution under an open-circuit condition. After a testing time of 200-300 h, SCC cracks grew to a certain size and the specimens were taken out. Each specimen was ultrasonically cleaned in deionized water as well as $5 \mathrm{wt} \% \mathrm{HCl}+2 \mathrm{~g} \mathrm{~L}^{-1}$ hexamethylenetetramine mixture. The two-dimensional morphology of the original surface containing an SCC crack was characterized by the scanning electron microscopy (SEM), and the crystallography was determined by the electron back-scattered diffraction (EBSD). Afterwards, the synchrotron-based X-ray computed tomography (XCT) was utilized to directly visualize three-dimensional morphologies of the SCC cracks. To perform tomographic imaging, $\sim 0.5$-mm-wide and -thick as well as $5-10-\mathrm{mm}$-long samples with the SCC cracks were prepared by the following method: wire electrode cutting of the cracked specimens; rinsing in acetone; cleaning in deionized water; hot air drying; and storing in a desiccated chamber. Due to the stress relaxation, the obtained SCC cracks occurred to open and widen from several microns to tens of microns. Subsequently, the computed tomographic experiments were conducted using the BL13W1 beamline at the Shanghai Synchrotron Radiation Facility. Almost monochromatic X-ray beam was used with the energy of $42 \mathrm{keV}$, and a high-speed camera recorded transmitted intensity in an $8 \mathrm{~s}$ exposure/projection interval, while the sample was rotated in $0.2^{\circ}$ increments. During each $180^{\circ}$ rotation, 900 two-dimensional radiographs were recorded and applied to the reconstruction of image slices nearly perpendicular to the crack growth direction. Isotropic voxels with the resolution of $0.6 \mu \mathrm{m}$ were achieved in the reconstructed slices. Next, image analysis, visualization, and three-dimensional rendering were carried out using a commercial software package (Amira).

\section{Crystallographic analysis of SCC cleavage planes}

The SCC cleavage planes were characterized according to the two-surface trace analysis. The Euler angles $\left(\varphi_{1}, \Phi, \varphi_{2}\right)$ of the original crystal plane $\left(h_{0}\right.$ 
$\left.k_{0} l_{0}\right)$ and the original crystal direction $\left[u_{0} v_{0} w_{0}\right]$ in Fig. 1 was $\left(317.8^{\circ}, 10.3^{\circ}\right.$, and $\left.18.9^{\circ}\right)$, characterized by the EBSD. The relation among the Euler angles, the original crystal plane and the original crystal direction is:

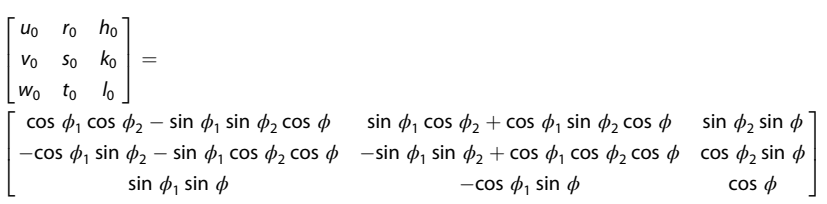

The detailed calculation shows that the original crystal plane $\left(h_{0} k_{0} I_{0}\right)$ and

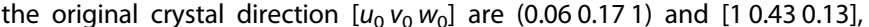
respectively. Further, the crystal direction [uvw] of the SCC cracks and the cleavage crystal planes $(h k l)$ can be calculated according to the twosurface trace analysis. For the crystal direction $[u v w]$, the crystallographic relations with the original crystal plane $\left(h_{0} k_{0} I_{0}\right)$ and the original crystal direction $\left[u_{0} v_{0} w_{0}\right]$ are described as Eqs. (17-18). That is,

$h_{0} u+k_{0} v+I_{0} w=0$

$\frac{u_{0} u+v_{0} v+w_{0} w}{\sqrt{u_{0}^{2}+v_{0}^{2}+w_{0}^{2}} \sqrt{u^{2}+v^{2}+w^{2}}}=\cos 60^{\circ}$ or $\cos 120^{\circ}$

Submitting $\left(h_{0} k_{0} I_{0}\right)=(0.060 .171)$ and $\left[u_{0} v_{0} w_{0}\right]=\left[\begin{array}{lll}1 & 0.430 .13\end{array}\right]$ into Eqs. $(17-18)$ yields $[u v w]=[1.2510 .25]$ or $[0.131-0.18]$. As for $(h k l)$ in Fig. 1c, the crystallographic relations with the crystal direction $[u v w]$ and the original crystal plane $\left(h_{0} k_{0} I_{0}\right)$ are correspondingly given by Eqs. (19-20):

$h u+k v+l w=0$

$\frac{h_{0} h+k_{0} k+I_{0} l}{\sqrt{h_{0}^{2}+k_{0}^{2}+l_{0}^{2}} \sqrt{h^{2}+k^{2}+l^{2}}}=\cos 103^{\circ}$ or $\cos 77^{\circ}$

The calculated result indicates that the cleavage crystal planes $(h k I)$ are $(4.9 \overline{0.46} 1),(\overline{4.1} 0.711),(\overline{1.7} \overline{1.8} 1)$, or $(10.313 .21)$. Since there are the minimal angle of $13^{\circ}$ between and $\left(\begin{array}{l}1 \\ 0\end{array}\right)$ with the lowest surface energy $y^{9,46}$, and certain errors when measuring the angles, the obtained cleavage planes of the SCC cracks in Fig. 1 is $\left(\begin{array}{ll}1 & 0\end{array}\right)$.

\section{First-principles simulations}

The face-centered cubic lattices of the FeCrNi alloy roughly equivalent to the type $316 \mathrm{~L}$ stainless steel were created using a 32 -atom periodic supercell model, in which the lattice sites were randomly occupied by $\mathrm{Fe}$, $\mathrm{Cr}$, and $\mathrm{Ni}$ atoms in an approximate ratio of 5:2:1 (see Supplementary Fig. 1). The slab with free top and bottom surfaces $\left(\begin{array}{lll}0 & 0 & 1\end{array}\right)$ consisted of 40 atoms in total, 6 atomic layers, and the surface vacuum layer over $20 \AA$ to avoid interactions with the periodic images, shown in Fig. 2a, d. In the case of hydrogen adsorption, $4 \mathrm{H}$ atoms uniformly located in the octahedral interstices on the top surface $(001)$, shown in Fig. $2 \mathrm{~b}$, e. The $\mathrm{H}$ - and $\mathrm{Cl}-$ adsorbed slab similarly contained $2 \mathrm{H}$ atoms and $2 \mathrm{Cl}$ atoms, shown in Fig. $2 \mathrm{c}$, f. At $0 \mathrm{~K}$ and on vacuum, the free surface energy of the 40-atom

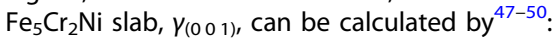

$\gamma_{(001)}=\frac{1}{2 A}\left(E_{\text {slab }}-25 \mu_{\mathrm{Fe}}-10 \mu_{\mathrm{Cr}}-5 \mu_{\mathrm{Ni}}\right)$

where $A$ is the surface area, $E_{\text {slab }}$ is the total free energy of the fully relaxed slab, $\mu_{\mathrm{Fe}}, \mu_{\mathrm{Cr}}$ and $\mu_{\mathrm{Ni}}$ separately represent the chemical potentials of $\mathrm{Fe}, \mathrm{Cr}$, and $\mathrm{Ni}$ atoms, and $25 \mu_{\mathrm{Fe}}+10 \mu_{\mathrm{Cr}}+5 \mu_{\mathrm{Ni}} \approx 40 / 32 E_{\text {supercell }}\left(E_{\text {supercell }}\right.$ is the total free energy of the 32 -atom supercell). As a consequence, $\gamma_{(0.1)}$ is given by:

$\gamma_{(001)}=\frac{1}{2 A}\left(E_{\text {slab }}-\frac{40}{32} E_{\text {supercell }}\right)$

Correspondingly, the $\mathrm{H}$-adsorbed surface energy of the 40 -atom $\mathrm{Fe}_{5} \mathrm{Cr}_{2} \mathrm{Ni}$ slab with $4 \mathrm{H}$ atoms, $\mathrm{Y}_{(001)}^{\mathrm{H}}$, is:

$\gamma_{(001)}^{\mathrm{H}}=\frac{1}{A}\left(E_{\text {slab }}^{\mathrm{H}}-\frac{40}{32} E_{\text {supercell }}-4 \mu_{\mathrm{H}}\right)-\gamma_{(001)}$

where $\mu_{\mathrm{H}}$ is the chemical potential of the $\mathrm{H}$ atom, approximating to the chemical potential of the $\mathrm{H}$ atom in the $\mathrm{H}_{2}$ molecule. The $\mathrm{H}$ - and $\mathrm{Cl}$ adsorbed surface energy of the 40 -atom $\mathrm{Fe}_{5} \mathrm{Cr}_{2} \mathrm{Ni}$ slab with $2 \mathrm{H}$ atoms and $2 \mathrm{Cl}$ atoms, $\mathrm{\gamma}_{(001)}^{\mathrm{H}+\mathrm{Cl}}$, is:

$\left.\gamma_{(001)}^{\mathrm{H}+\mathrm{Cl}}=\frac{1}{A}\left(E_{\text {slab }}^{\mathrm{H}+\mathrm{Cl}}-\frac{40}{32} E_{\text {supercell }}-2 \mu_{\mathrm{H}}-2 \mu_{\mathrm{Cl}}\right)-\gamma_{(0} 01\right)$ where $\mu_{\mathrm{Cl}}$ is the chemical potential of the $\mathrm{Cl}$ atom, approximating the chemical potential of the $\mathrm{Cl}$ atom in the $\mathrm{Cl}_{2}$ molecule. The structures of the supercell, slabs, and molecules were all optimized through the DFT as implemented in the VASP code ${ }^{51-53}$. The energy and the force converged to $10^{-5}$ and $10^{-2} \mathrm{eV} \AA^{-1}$, respectively.

\section{DATA AVAILABILITY}

All data that support the findings of this study are available from the corresponding author upon reasonable request.

Received: 12 August 2020; Accepted: 7 April 2021; Published online: 20 May 2021

\section{REFERENCES}

1. Logan, H. L. Film-rupture mechanism of stress corrosion. J. Res. Natl. Bur. Stand. 48, 99-105 (1952)

2. Qiao, L. J., Mao, X. \& Luo, J. L. Micromechanics of stress corrosion cracking of single-crystal austenitic type 321 stainless steel under mode II loading. Corrosion 52, 927-933 (1996).

3. Li, J. X., Chu, W. Y., Wang, Y. B. \& Qiao, L. J. In situ TEM study of stress corrosion cracking of austenitic stainless steel. Corros. Sci. 45, 1355-1365 (2003).

4. Wenman, M. R., Trethewey, K. R., Jarman, S. E. \& Chard-Tuckey, P. R. A finiteelement computational model of chloride-induced transgranular stress-corrosion cracking of austenitic stainless steel. Acta Mater. 56, 4125-4136 (2008).

5. Woodtli, J. \& Kieselbach, R. Damage due to hydrogen embrittlement and stress corrosion cracking. Eng. Fail. Anal. 7, 427-450 (2000).

6. Qiao, L. J., Gao, K. W. \& Luo, J. L. Discontinuous surface cracks during stress corrosion cracking of stainless steel single crystal. Corros. Sci. 53, 3509-3514 (2011).

7. Zhu, L. K., Yan, Y., Qiao, L. J. \& Volinsky, A. A. Stainless steel pitting and early-stage stress corrosion cracking under ultra-low elastic load. Corros. Sci. 77, 360-368 (2013).

8. Zhu, L. K., Yan, Y., Li, J. X., Qiao, L. J. \& Volinsky, A. A. Stress corrosion cracking under low stress: continuous or discontinuous cracks? Corros. Sci. 80, 350-358 (2014).

9. Zhu, L. K. et al. Stress corrosion cracking at low loads: Surface slip and crystallographic analysis. Corros. Sci. 100, 619-626 (2015).

10. Zhu, L. K., Li, Z. C. \& Qiao, L. J. A three-dimensional model of non-slipping stress corrosion cracking under low loads. Struct. Integr. Procedia 2, 612-621 (2016).

11. Newman, R. C. \& Healey, C. Stability, validity and sensitivity to input parameters of the slip-dissolution model for stress-corrosion cracking. Corros. Sci. 49, 4040-4050 (2007).

12. Vermilyea, D. A. A theory for the propagation of stress corrosion cracks in metals. J. Electrochem. Soc. 119, 405-407 (1972).

13. Kolman, D. G. \& Scully, J. R. Continuum mechanics characterization of plastic deformation-induced oxide film rupture. Philos. Mag. A 79, 2313-2338 (1999).

14. Alyousif, O. M. \& Nishimura, R. The stress corrosion cracking behavior of austenitic stainless steels in boiling magnesium chloride solutions. Corros. Sci. 49, 3040-3051 (2007)

15. Gutman, E. M. An inconsistency in 'film rupture model' of stress corrosion cracking. Corros. Sci. 49, 2289-2302 (2007).

16. Magnin, T., Chieragatti, R. \& Oltra, R. Mechanism of brittle fracture in a ductile 316 alloy during stress corrosion. Acta Metall. Mater. 38, 1313-1319 (1990).

17. Magnin, T., Chambreuil, A. \& Bayle, B. The corrosion-enhanced plasticity model for stress corrosion cracking in ductile fcc alloys. Acta Mater. 44, 1457-1470 (1996).

18. Chateau, J. P., Delafosse, D. \& Magnin, T. Numerical simulations of hydrogen dislocation interactions in fcc stainless steels: part Il: hydrogen effects on crack tip plasticity at a stress corrosion crack. Acta Mater. 50, 1523-1538 (2002).

19. Flanagan, W. F., Bastias, P. \& Lichter, B. D. A theory of transgranular stress corrosion cracking. Acta Metall. Mater. 39, 695-705 (1991).

20. Chateau, J. P., Delafosse, D. \& Magnin, T. Numerical simulations of hydrogen dislocation interactions in fcc stainless steels: part I: hydrogen-dislocation interactions in bulk crystals. Acta Mater. 50, 1507-11522 (2002).

21. Masuda, H. SKFM observation of SCC on SUS304 stainless steel. Corros. Sci. 49, 120-129 (2007).

22. Arnoux, P. Atomistic simulations of stress corrosion cracking. Corros. Sci. 52, 1247-1257 (2010).

23. Liu, H. W. A unified model of environment-assisted cracking. Acta Mater. 56, 4339-4348 (2008).

24. Tang, Y. \& El-Awady, J. A. Atomistic simulations of the interactions of hydrogen with dislocations in fcc metals. Phys. Rev. B 86, 174102 (2012). 
25. Gu, Y. J. \& El-Awady, J. A. Quantifying the effect of hydrogen on dislocation dynamics: a three-dimensional discrete dislocation dynamics framework. J. Mech. Phys. Solids 112, 491-507 (2018).

26. Zhang, B. et al. Unmasking chloride attack on the passive film of metals. Nat. Commun. 9, 1-9 (2018).

27. Qiao, L. J. \& Mao, X. Thermodynamic analysis on the role of hydrogen in anodic stress corrosion cracking. Acta Metall. Mater. 34, 4001-4005 (1995).

28. Wang, W. W. et al. The role of hydrogen in stress corrosion cracking of 310 austenitic stainless steel in a boiling $\mathrm{MgCl}_{2}$ solution. Corros. Sci. 60, 275-279 (2012).

29. Liu, H. W. Reply to comments on 'A unified model of environment assisted cracking'. Scr. Mater. 61, 335-337 (2009).

30. Chen, Y.-S. et al. Observation of hydrogen trapping at dislocations, grain boundaries, and precipitates. Science 367, 171-175 (2020).

31. Zamora, R. J., Nair, A. K., Hennig, R. G. \& Warner, D. H. Ab initio prediction of environmental embrittlement at a crack tip in aluminum. Phys. Rev. B 86, 060101 (2012).

32. Sieradzki, K. \& Newman, R. C. Brittle behavior of ductile metals during stress corrosion cracking. Philos. Mag. A 51, 95-132 (1985).

33. Zhang, C., Su, Y. J., Qiao, L. J. \& Chu, W. Y. Tarnishing film-induced brittle cracking of brass. J. Mater. Res. 24, 2409-2415 (2009).

34. Guo, T., Qiao, L. J., Pang, X. L. \& Volinsky, A. A. Brittle film-induced cracking of ductile substrates. Acta Mater. 99, 273-280 (2015).

35. Chu, W. Y., Qiao, L. J., Chen, Q. Z. \& Gao, K. W. Fracture and Environmental Assisted Cracking (Science Press, 2003).

36. Chu, W. Y. et al. Hydrogen Embrittlement and Stress Sorrosion Cracking (Science Press, 2013).

37. Bitzek, E., Kermode, J. R. \& Gumbsch, P. Atomistic aspects of fracture. Inter. J. Fract. 191, 13-30 (2015).

38. Lawn, B. Fracture of Brittle Solids (Cambridge University Press and Higher Education Press, 2009).

39. Adamson, A. W. Physical Chemistry of Surfaces (Wiley, 1990).

40. Bermúdez-Salguero, C. \& Gracia-Fadrique, J. Analysis of Gibbs adsorption equation and thermodynamic relation between Gibbs standard energies of adsorption and micellization through a surface equation of state. J. Colloid Interface Sci. $\mathbf{3 5 5}$, 518-519 (2011).

41. Liu, R., Narita, N., Altstetter, C., Birnbaum, H. \& Pugh, E. N. Studies of the orientations of fracture surfaces produced in austenitic stainless steels by stress-corrosion cracking and hydrogen embrittlement. Metall. Trans. A 11, 1563-1574 (1980).

42. Meletis, E. I. \& Hochman, R. F. The crystallography of stress corrosion cracking in face centered cubic single crystals. Corros. Sci. 24, 843-862 (1984).

43. Meletis, E. I. \& Hochman, R. F. A review of the crystallography of stress corrosion cracking. Corros. Sci. 26, 63-90 (1986). 77, 79.

44. Li, S., Dickson, J. I. \& Bailon, J. P. The influence of the stress intensity factor on the fractography of stress corrosion cracking of 316 stainless steel. Mater. Sci. Eng. A 119, 59-72 (1989).

45. Dickson, J. I., Groulx, D. \& Li, S. The fractography of stress corrosion cracking of 310 stainless steel: crystallographic aspects and the influence of stress intensity factor. Mater. Sci. Eng. A 94, 155-173 (1987).

46. Caglioti, G., Rizzi, G. \& Bilello, J. C. Surface energy for brittle fracture in metals from phonon frequencies. J. Appl. Phys. 42, 4271-4276 (1971).

47. Rapcewicz, K., Chen, B., Yakobson, B. \& Bernholc, J. Consistent methodology for calculating surface and interface energies. Phys. Rev. B 57, 7281 (1998).

48. Zhang, W. \& Smith, J. R. Stoichiometry and adhesion of $\mathrm{Nb} / \mathrm{Al}_{2} \mathrm{O}_{3}$. Phys. Rev. B 61 16883 (2000)

49. Siegel, D. J., Hector, L. G. Jr. \& Adams, J. B. Adhesion, stability, and bonding at metal/metal-carbide interfaces: Al/WC. Surf. Sci. 498, 321-336 (2002).
50. Liu, P.-L. \& Siao, Y.-J. Ab initio study on preferred growth of ZnO. Scr. Mater. 64, 483-485 (2011).

51. Kresse, G. \& Furthmüller, J. Efficient iterative schemes for ab initio total-energy calculations using a plane-wave basis set. Phys. Rev. B 54, 11169 (1996).

52. Kresse, G. \& Hafiner, J. Ab initio molecular dynamics for liquid metals. Phys. Rev. $B$ 47, 558 (1993).

53. Kresse, G. \& Joubert, D. From ultrasoft pseudopotentials to the projector augmented-wave method. Phys. Rev. B 59, 1758 (1999).

\section{ACKNOWLEDGEMENTS}

This work is supported by the National Natural Science Foundation of China under grant 51901024. We would like to acknowledge the experimental help with the synchrotron-based X-ray computed tomography by the BL13W1 beamline at the Shanghai Synchrotron Radiation Facility.

\section{AUTHOR CONTRIBUTIONS}

L.Z. conceived the experimental design and created the cleavage-dissolution model. Y. Li performed the first-principles simulations.

\section{COMPETING INTERESTS}

The authors declare no competing interests.

\section{ADDITIONAL INFORMATION}

Supplementary information The online version contains supplementary material available at https://doi.org/10.1038/s41529-021-00172-7.

Correspondence and requests for materials should be addressed to L.Z.

Reprints and permission information is available at http://www.nature.com/ reprints

Publisher's note Springer Nature remains neutral with regard to jurisdictional claims in published maps and institutional affiliations.

Open Access This article is licensed under a Creative Common Attribution 4.0 International License, which permits use, sharing, adaptation, distribution and reproduction in any medium or format, as long as you give appropriate credit to the original author(s) and the source, provide a link to the Creative Commons license, and indicate if changes were made. The images or other third party material in this article are included in the article's Creative Commons license, unless indicated otherwise in a credit line to the material. If material is not included in the article's Creative Commons license and your intended use is not permitted by statutory regulation or exceeds the permitted use, you will need to obtain permission directly from the copyright holder. To view a copy of this license, visit http://creativecommons. org/licenses/by/4.0/.

(c) The Author(s) 2021 